\title{
Detailed particle-in-cell simulations on the transport of a relativistic electron beam in plasmas
}

\author{
Anupam Karmakar, ${ }^{*}$ Naveen Kumar, ${ }^{\dagger}$ and Alexander Pukhov \\ Institut für Theoretische Physik I, Heinrich-Heine-Universität, Düsseldorf 40225, Germany \\ O. Polomarov and Gennady Shvets \\ Department of Physics and Institute for Fusion Studies, University of Texas at Austin, One University Station, Austin, Texas 78712, USA
}

(Received 18 July 2008; revised manuscript received 26 February 2009; published 2 July 2009)

\begin{abstract}
We present comprehensive two-dimensional (2D) particle-in-cell (PIC) simulations on the transport of a relativistic electron beam in a plasma in the context of fast ignition fusion. The 2D PIC simulations are performed by constructing two different simulation planes and have shown the complete stabilization and destabilization of the Weibel instability due to the beam temperature and background plasma collisions, respectively. Some three-dimensional PIC simulation results on the filamentary structures are also shown thereby further shedding light on the filamentation of the electron beam in plasmas. The linear growth rates of fastest growing mode in the beam-plasma system are compared with a theoretical model developed and are found in good agreement with each other.
\end{abstract}

DOI: 10.1103/PhysRevE.80.016401

PACS number(s): 52.57.-z, 52.35.-g, 52.65.Rr

\section{INTRODUCTION}

Transportation of a very high current electron beam through overdense plasmas is a key problem in several areas especially in fast ignition fusion (FI). In the FI scheme, a laser-generated relativistic electron beam with a few $\mathrm{MeV}$ per electron energy must propagate through an overdense plasma to heat a hot spot in the core of a precompressed fusion fuel target [1]. The current carried by these MeV electrons inside the plasma is much higher than the Alfvén current limit $I=\left(m c^{3} / e\right) \gamma=17 \gamma \mathrm{kA}$, where $m$ is the electron mass, $e$ is the electronic charge, and $\gamma$ is the Lorentz factor of the beam. Clearly, the transportation of this electron beam is not possible unless it is compensated by a return plasma current, thus maintaining the global charge neutrality. However, this configuration, where two beams are counterpropagating, is unstable and the current beams are susceptible to the Weibel (also referred to as the filamentation) and twostream instabilities. The Weibel instability [2] is an aperiodic instability in collisionless plasmas and it produces strong quasistatic magnetic field in the system. Huge magnetic field associated with it has been observed in particle-in-cell (PIC) simulations and in experiments in laser-plasma interaction [3]. The Weibel instability is also considered to be the main mechanism of the huge magnetic-field generation in the collision of two plasma shells, which is also known as gamma ray bursts (GRBs) in astrophysics. During the GRBs, strong radiation is emitted which is believed to be of the synchrotron origin, requiring the presence of huge quasistatic magnetic fields at first place, thus necessitating the need of invoking the Weibel instability in astrophysical problems [4]. In FI, the Weibel instability breaks the relativistic electron

\footnotetext{
*Present address: Institute for Advanced Simulation, Jülich Supercomputing Centre, Forschungszentrum Jülich GmbH, D-52425, Germany and EMMI, GSI, Darmstadt, Germany; a.karmakar@fzjuelich.de

${ }^{\dagger}$ kumar@tp1.uni-duesseldorf.de
}

beam into tiny filaments and can severely affect the energy deposition in the core of the compressed fusion pellet. It has been a subject of research for a long time [5-17]. Lee and Lampe [5] first presented seminal PIC simulation results on the Weibel instability in FI plasmas. Yoon and Davidson [8] have developed a kinetic formalism of the Weibel instability in the context of charged particle beam transport in accelerator physics. Recently, various theoretical models, both kinetic and hydrodynamic ones, have been developed to study this instability in linear and quasilinear regimes [9-11]. Honda et al. [12] have studied the collective stopping of the beam and ion heating in the context of FI. Three-dimensional (3D) simulations of resistive beam filamentation corresponding to the full scale FI configuration have been performed [14]. Three-dimensional magnetic structures generated due to the Weibel instability in a collisionless plasma have also been reported [15]. Recently, the evidence of Weibel-type dynamics and the resultant filamentation of electron beams have been reported experimentally [17].

As pointed out earlier, in 3D geometry the electron beam is actually susceptible to the Weibel and two-stream instabilities. Though the Weibel instability can be suppressed by the transverse beam temperature [7], the two-stream instability is unaffected by the transverse beam temperature. Hence, in 3D geometry we may still have filamentation of the beam irrespective of the transverse beam temperature. Until now PIC simulations have been limited to the study of either the Weibel instability in 2D geometry or the coupled Weibel-twostream instability in 3D geometry [12-16]. Thus they have not separated the role of these instabilities on the filamentation of the electron beam. Moreover, these studies also did not distinctly examine the influence of various factors such as beam temperature, collisions in return plasma current on the filamentation of the electron beam.

In this paper, we study the filamentation of the electron beam and present the results of 2D and 3D PIC simulations on the transport of a relativistic electron beam in overdense plasmas. Two different simulation geometries for the 2D simulations are constructed: (1) transverse geometry, which is a plane perpendicular to the direction of the beam propa- 
gation direction and (2) longitudinal geometry, a plane containing the propagating beam. The rationale behind the construction of these simulation geometries is borne out of the fact that in transverse geometry the coupling of the Weibel instability with the two-stream instability does not occur, whereas the longitudinal geometry is appropriate to study the coupling of the Weibel instability with the two-stream instability. Thus, these two geometries can separate the roles of the Weibel and two-stream instabilities on the filamentation of the electron beam. The effect of the background plasma collisions and the beam temperature on the beam filamentation dynamics is studied in an intuitive way by choosing four different simulation runs: (a) cold electron beam and collisionless background plasmas, (b) cold electron beam and background collisional plasma, (c) warm electron beam and background collisionless plasma, and (d) warm electron beam and background collisional plasma. Hence, we have studied the filamentation of the relativistic beam extensively highlighting the role of each physical process clearly. The simulation results show that the Weibel instability cannot be suppressed by thermal effects only, if small collisions are present in the background plasma. We have performed further full 3D simulations to study the filamentation of the electron beam. These will be presented here briefly. The remainder of the paper is organized as follows: the simulation geometries and results of 2D PIC simulations are presented in Sec. II. We also briefly present the results of 3D PIC simulations in Sec. III. In Sec. IV, we derive the dispersion relations for the simulation geometries and estimate the linear growth rate of the most unstable mode in different simulation geometries. In Sec. V, we conclude our results.

\section{2D PIC SIMULATIONS}

In 2D simulations, we choose two different simulation planes namely transverse and longitudinal geometries. In transverse geometry, the simulation domain is decomposed in $(X \times Y)$ Cartesian plane. The electron beam propagates in negative $\hat{z}$ direction with relativistic velocity $v_{b z} \gg v_{b x}, v_{b y}$. The cold background plasma is represented by ambient plasma electrons and flows opposite to the beam electrons with the velocity $v_{p z}$ to compensate the beam charge. The plasma ions are considered as a fixed charge-neutralizing background with the density $n_{0}=n_{b}+n_{p}$. In longitudinal geometry, the simulation domain consists of $(X \times Y)$ plane and the electron beam propagates along the negative $\hat{x}$ direction with relativistic velocity $v_{b x} \gg v_{b y}, v_{b z}$. The beam density is much smaller than the background plasma electron density, i.e., $n_{b} \ll n_{p}$, which is a usual situation in FI. The spatial dimensions of the simulation domain $L$ are large in comparison with the plasma skin depth, i.e., $L \gg \lambda_{s}$, where $\lambda_{s}$ $=c / \omega_{p}$, where $c$ and $\omega_{p}$ are the velocity of light in vacuum and the total electron plasma frequency, respectively. The quasineutrality is maintained overall as the field evolutions occur on a time scale slower than the electron plasma frequency $\Delta t \gg 1 / \omega_{p}$. The collisional processes are simulated with a newly implemented collision module in the relativistic PIC code Virtual Laser Plasma Laboratory (VLPL) [18]. The module is based on the stochastic scattering of the plasma particles employing scattering cross sections.
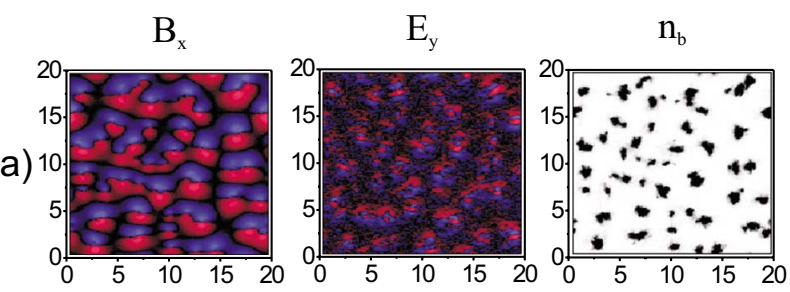

b)
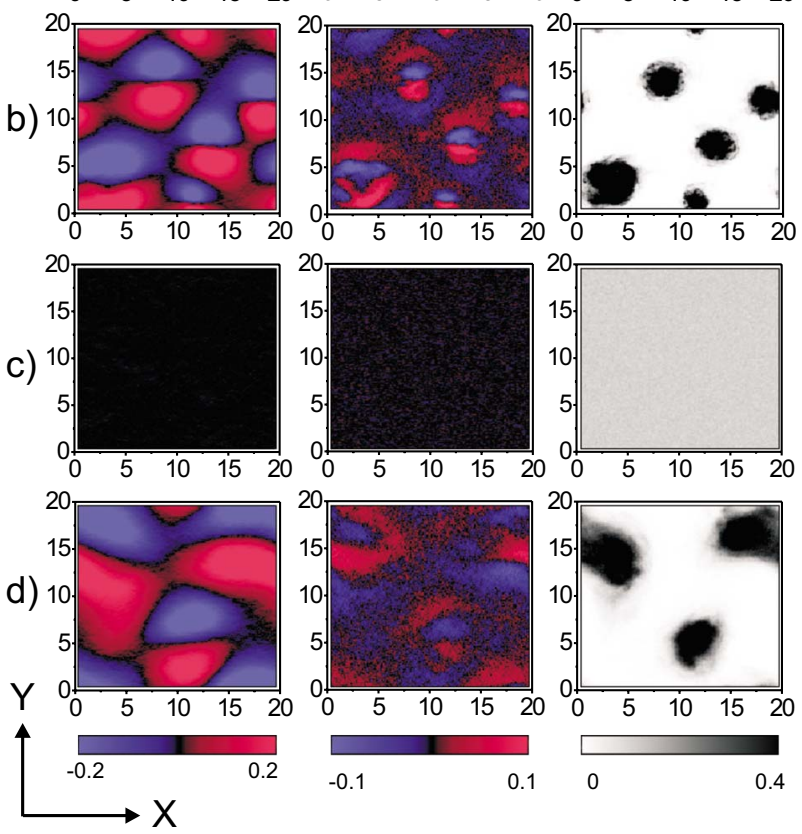

FIG. 1. (Color online) Snapshots of the transverse electromagnetic fields $\left(B_{x}\right.$ and $\left.E_{y}\right)$ and beam filaments in transverse geometry at a time $T=20\left(2 \pi / \omega_{p}\right)$ for four different simulation cases: (a), (b), (c), and (d). The horizontal and vertical axes represent the simulation domain as described in the text.

The simulation planes have dimensions, $(X \times Y)=20 \lambda_{s}$ $\times 20 \lambda_{s}$, and are sampled with a mesh of $160 \times 160$ cells. All simulations are performed with 64 particles per cell and with a spatial resolution smaller than the skin depth $\delta x=\delta y$ $=0.125 \lambda_{s}$. The density ratio between the plasma and beam electrons is $n_{p} / n_{b}=9$, whereas the beam and the background plasma electrons have velocities $v_{b}=0.9 c$ and $v_{p}=-0.1 c$, respectively. The density ratio chosen is a typical example of a scenario in which the electron beam is interacting with the outer surface of the pallet in FI. The evolution of every component $F_{i}$ of the fields $\mathbf{E}$ and $\mathbf{B}$ is recorded at every diagnostic step summed over all the particles as $\int_{S}\left(e F_{i} / m_{e} c \omega_{p}\right)^{2} h_{x} h_{y}$, where $h_{x}$ and $h_{y}$ are the spatial grid steps along $x$ and $y$, and $e F_{i} / m_{e} c \omega_{p}$ represents the field normalization. The electron beam has a temperature of $T_{b} \approx 70 \mathrm{keV}$ and the ambient plasma collision frequency $\nu_{e i} / \omega_{p}=0.15$ is used for these simulations. In all simulations, the background plasma is always cold while the beam electrons do not face any collisions.

\section{A. Transverse geometry}

In Fig. 1, the snapshots of transverse $\mathbf{E}$ and $\mathbf{B}$ fields and the structure of the beam filaments are shown at a time $T$ $=20\left(2 \pi / \omega_{p}\right)$ for four different simulation cases. The beam 


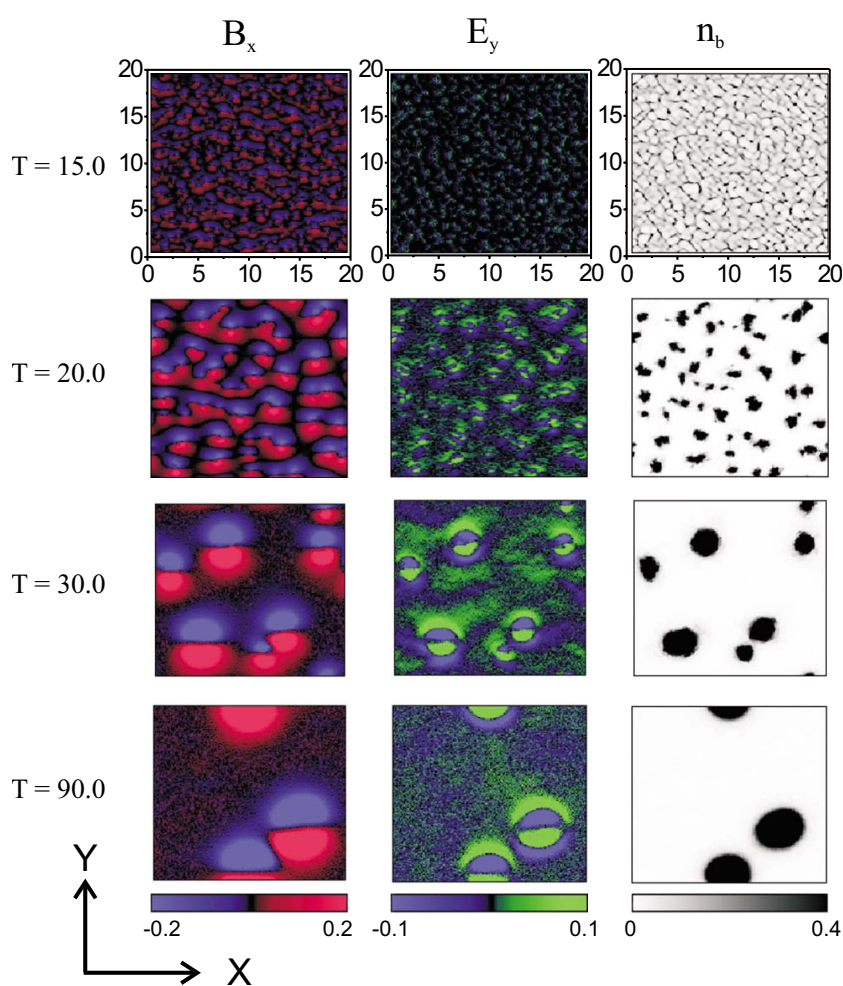

FIG. 2. (Color online) Temporal evolution of electromagnetic fields $\left(B_{x}\right.$ and $\left.E_{y}\right)$ and beam filaments at different times in transverse geometry for the simulation case (a).

density filamentation is shown in the last column in each panel. In the case (a), the filaments are small, comparable with the plasma skin depth. In the case (b), the filament size is bigger as the collective effects of plasma (collisional diffusion of plasma electrons across the self-generated magnetic fields) come into effect. In the third panel of the figure, simulation case $(\mathrm{c})$, the electron beam has transverse temperature $T_{b} \sim 70 \mathrm{keV}$ and the background plasma electrons are collisionless; we see no filament formation. The temperature of the electron beam stabilizes the Weibel instability. Physically the thermal pressure of the electron beam prevails over the magnetic pressure in this case. Hence, the magnetic field pinching that actually drives the instability does not occur resulting in the suppression of the Weibel instability. The last panel of figure depicts the filament formation in the simulation case (d). One remarkable result is that although the beam temperature is the same as in the stable collisionless case (c), the background plasma collisions revive back the instability. The role played by the collisions here is somewhat paradoxical as one might expect that the collective effects of both collisions and temperature must suppress the instability. Yet, quite opposite is the case. This happens due to the collision-induced negative energy waves generation in the beam-plasma system, which drives the filamentation of the beam. This is discussed later in the paper. Figure 2 shows the temporal evolution of the filaments merging and fields evolution for the case (a).

Figure 3 shows the evolution of electric- and magneticfield energies in the four cases corresponding to the simulations in Fig. 1. We wish to state here that in the simulations
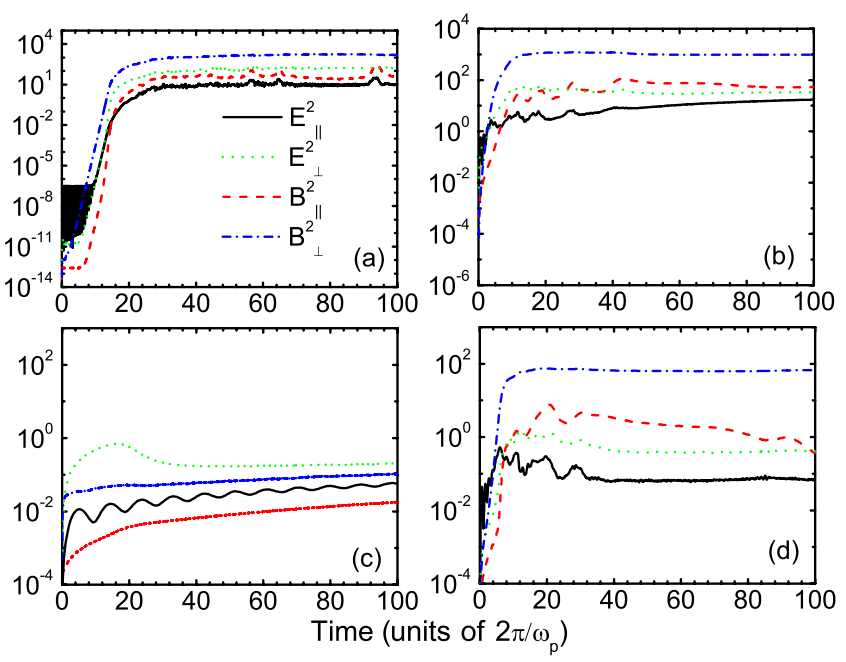

FIG. 3. (Color online) Temporal evolution of the field energies $\left(E_{\perp}^{2}, E_{\|}^{2}, B_{\perp}^{2}\right.$, and $\left.B_{\|}^{2}\right)$ in the transverse geometry for four different simulation cases as in Fig. 1. In all subplots, the vertical axes represent normalized field energy components in logarithmic scales as described in text, whereas the horizontal axes show the time normalized by $2 \pi / \omega_{p}$.

the generated electric- and magnetic-field energies are a small fraction of the initial beam kinetic energy. Hence, care should be taken about the energy conservation issue in PIC simulations as even small numerical errors in simulations can produce unphysical field energy buildup. The energy axes in Fig. 3 are in logarithmic scales. We see a stage of linear instability, where the field energies build up exponentially in time. It is followed by the nonlinear saturation of the instability. The linear instability stage is present in the simulations (a), (b), and (d). The simulation case (c) shows no linear instability and no significant buildup of the field energy. This is in compliance with the results of Fig. 1. After the linear stage of the instability, filaments start merging due to the magnetic attraction and the field energies saturate. Some small fluctuations around the saturated field energies can be seen. A magnified look into these fluctuations, corresponding to the collective merging of the beam filaments in the saturation regime, is shown in Fig. 4. We observe a surge in the $E_{z}$ field whenever the filaments merge. The merging of bigger filaments produces very large fluctuation in the field energy, also discussed by Honda et al. [12].

\section{B. Longitudinal geometry}

The snapshots of transverse $\mathbf{E}$ and $\mathbf{B}$ fields and the structure of the beam filaments for longitudinal geometry are shown in Fig. 5 for all four different simulation cases as in Sec. II A. The beam density filamentation is shown in the extreme right column in each panel. The filament pattern differs significantly in this case due to the presence of coupled instability. The filamentation process constructs pipelike structures and the beam electrons get diffused leading to the field saturation. In the case (a), the filamentation occurs at a slower rate and the field energies reach the saturation level at later times than the other three cases. Hence, 


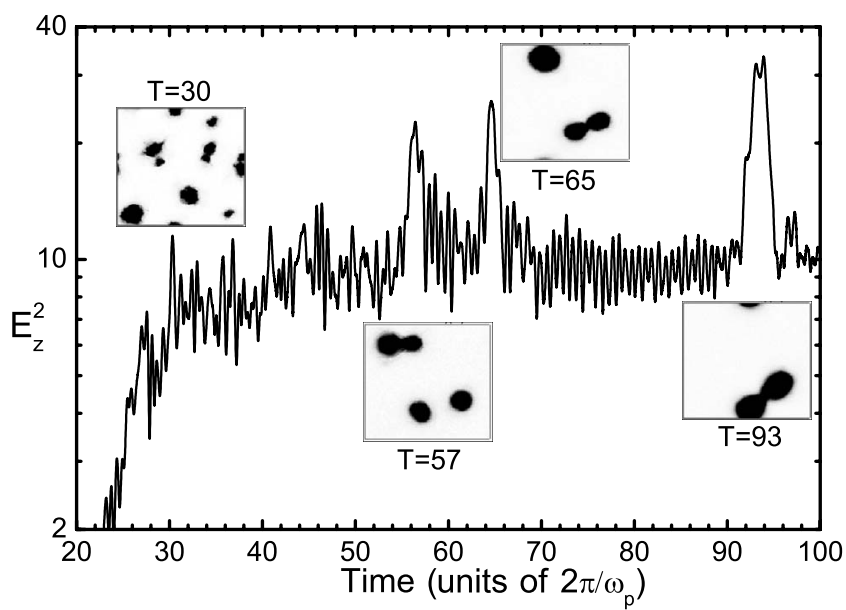

FIG. 4. Fluctuations in the longitudinal electric-field energy $E_{z}^{2}$ corresponding to collective merging of the filaments in the saturation regime. The insets show the merging of the filaments by a corresponding fluctuation in the field energy.

the snapshots for the case (a) is taken at $T=15\left(2 \pi / \omega_{p}\right)$ and the snapshots in the next three panels are at a different time $T=8\left(2 \pi / \omega_{p}\right)$. The filaments in the case (a) are small and comparable to the background plasma electron skin depth,

a)

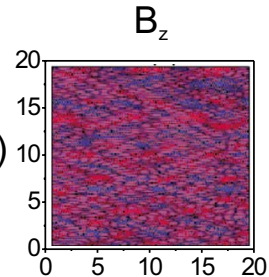

b)

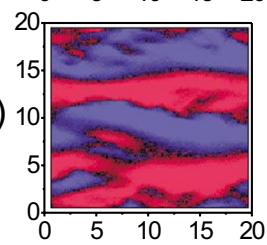

C)
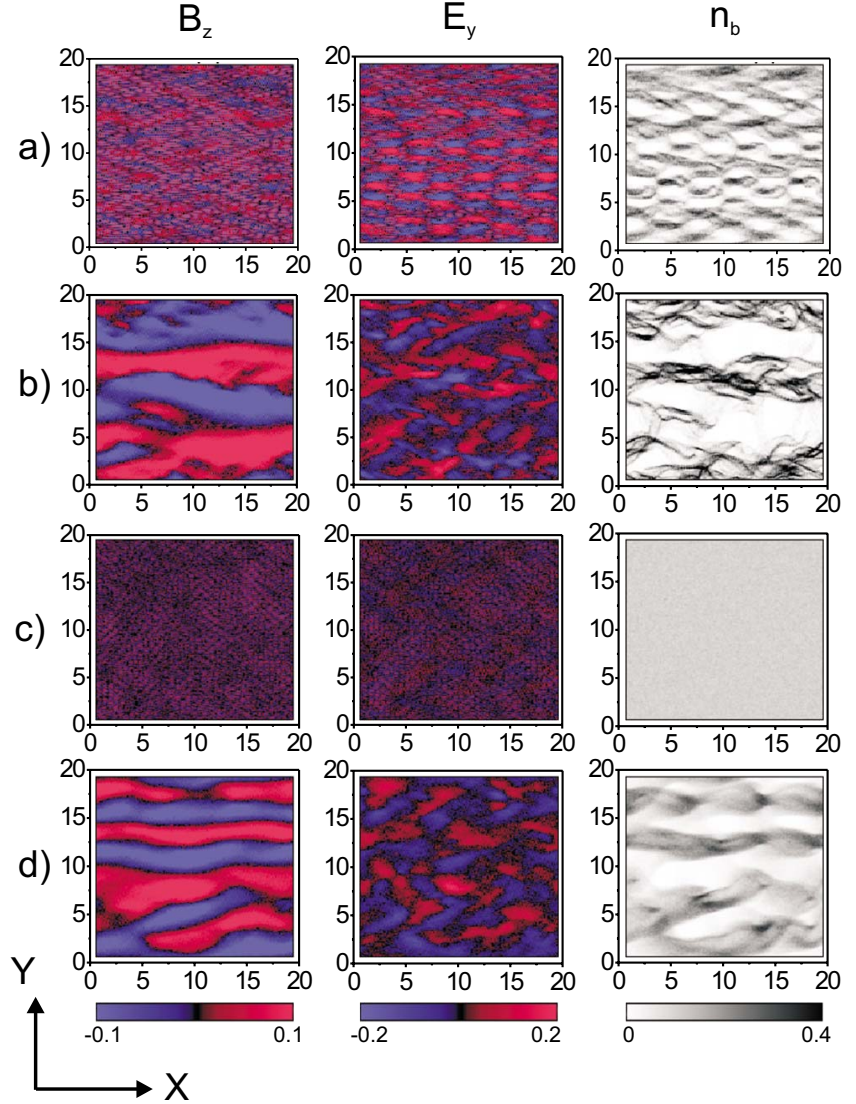

FIG. 5. (Color online) Snapshots of the electromagnetic fields $\left(B_{z}\right.$ and $\left.E_{y}\right)$ and beam filaments in longitudinal geometry for four different simulation cases as in Fig. 1. The top most panel, i.e., in case (a), is taken at a time $T=15\left(2 \pi / \omega_{p}\right)$ and the other three are taken at times $T=8\left(2 \pi / \omega_{p}\right)$.

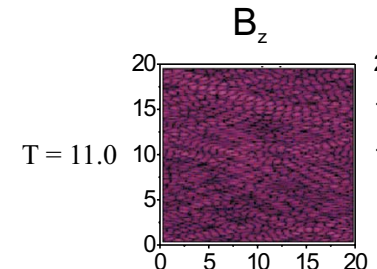

$E_{x}$
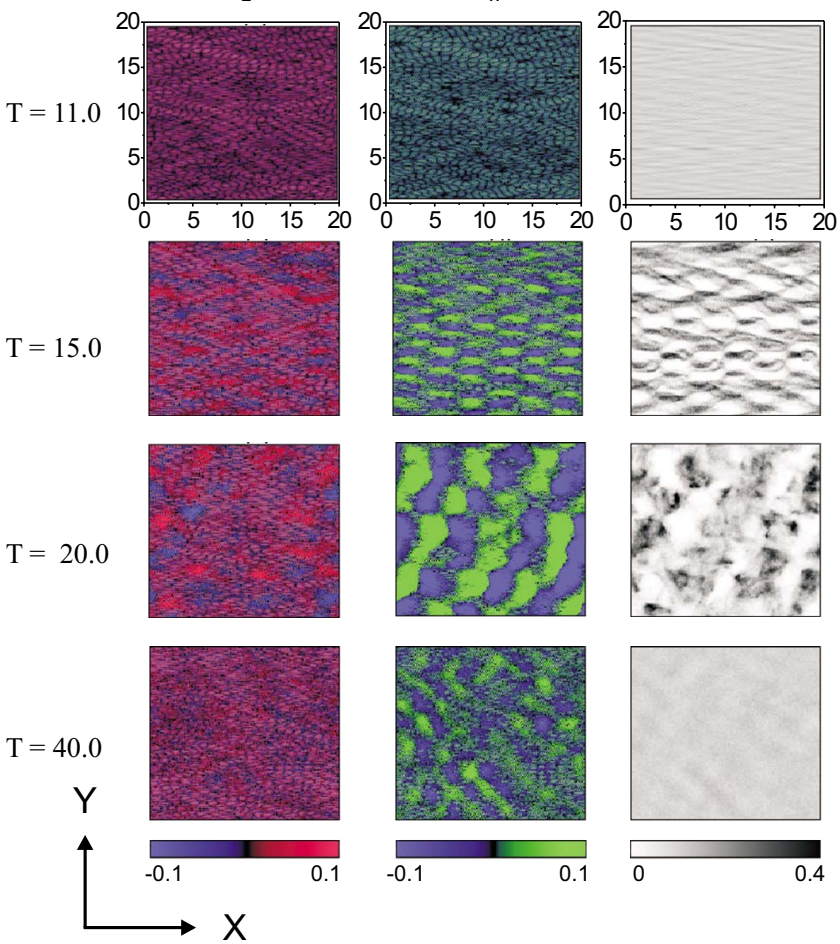

FIG. 6. (Color online) Temporal evolution of the beam filaments $\left(n_{b}\right)$ in longitudinal geometry for the simulation case (a).

likewise the transverse case. In the case (b), the filament size is bigger and the extent of electron diffusion is broader. One expects a lower buildup of the field energies than the previous case in the presence of collisions, which is also seen from the simulation results here. The third panel of Fig. 5 shows the simulation case (c) and one does not see any filament formation similar to the transverse geometry case, but evolution of the field energies can be noticed in Fig. 7. Although the Weibel instability, which is largely responsible for filament formation in relativistic conditions, gets suppressed the two-stream instability still persists and contributes to the buildup of field energies in the system. The two-stream instability results in large-scale filament formation of the beam which is not clearly visible in the simulation case (c). (These filaments can be observed in 3D PIC simulations in the next section.) The last panel of the figures depicts the filament formation in the simulation case (d), where electron beam is hot and the background plasma is collisional. Figure 6 shows the temporal development for the filament merging process for the case (a).

Figure 7 shows the evolution of the electric- and magnetic-field energies for the four cases in the longitudinal geometry. Likewise to the transverse geometry, one notices a stage of linear instability where the field energies build up exponentially in time, which is then followed by nonlinear saturation. Unlike the transverse geometry, the linear instability stage is present in all the simulations (a)-(d). A very small growth of the fields is observed in the simulation (c), where the electron beam has high temperature and the background plasma is collisionless. This small growth rate results due to the presence of unsuppressed two-stream instability. 

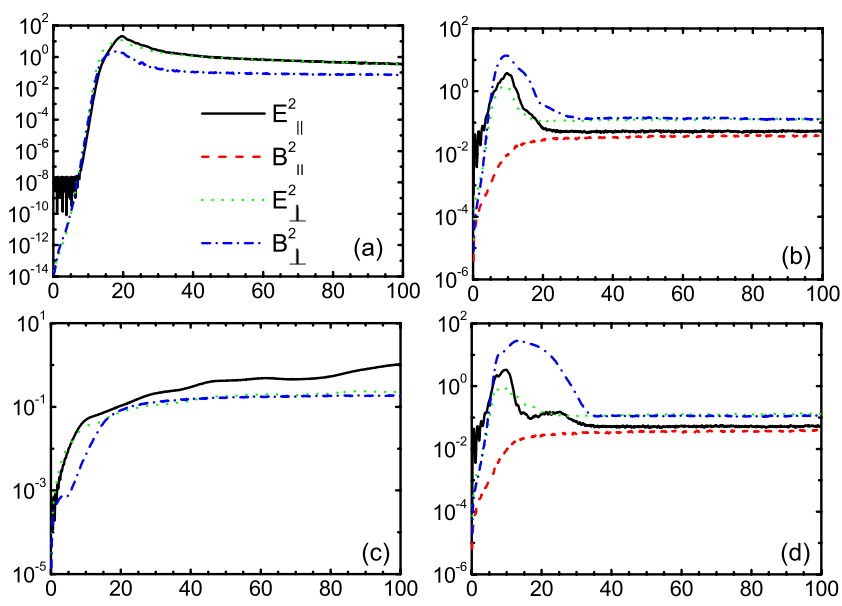

FIG. 7. (Color online) Time evolution of the field energies $\left(E_{\perp}^{2}\right.$, $B_{\perp}^{2}, E_{\|}^{2}$, and $\left.B_{\|}^{2}\right)$ of longitudinal geometry for four different simulation cases as described in Fig. 1. The axes in subplots are the same as in Fig. 3.

Nevertheless, the field energies saturate rapidly and do not grow to higher magnitudes as in the other three cases. This is in compliance with the results of Fig. 1. Afterward, during the nonlinear stage of the instability, the filaments merge rapidly due to the magnetic attraction and the field energies saturate. We also see strong diffusion of the beam electrons during the merging process. In the cases (b) and (d), this diffusion prevails more widely and the saturation occurs much earlier than the collisionless cases. The drop in the fields $E_{\|}, E_{\perp}$, and $B_{\perp}$ in (b) and (d) can be explained due to the dominance of collisional diffusion of the beam electrons over merging of the filament structures. Moreover, a growth of the longitudinal magnetic field $B_{\|}$(broken red lines) also occurs due to collisions in the system.

\section{3D PIC SIMULATIONS}

We have also carried out full 3D simulations to fully understand the structure of the filaments and fields. For 3D simulations, the simulation box dimensions are $X \times Y \times Z$

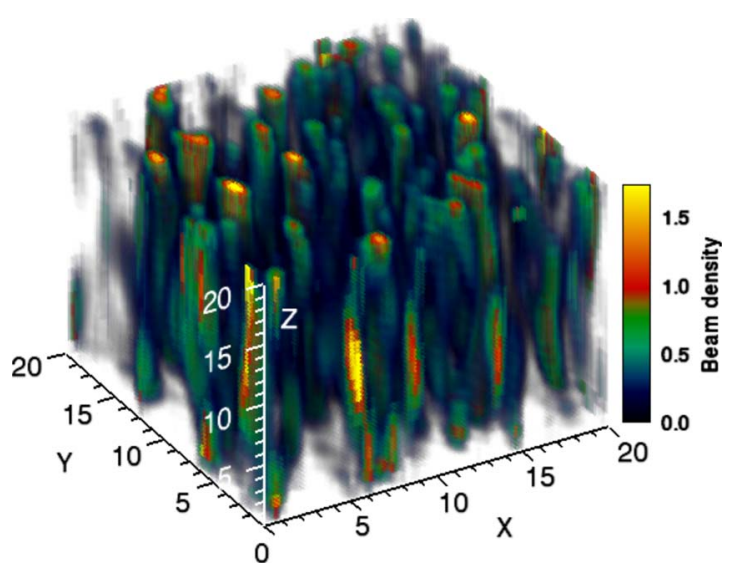

FIG. 8. (Color online) 3D filamentary structures for the case of the cold electron beam and collisionless background plasma, i.e., case (a), at a time $T=16\left(2 \pi / \omega_{p}\right)$.

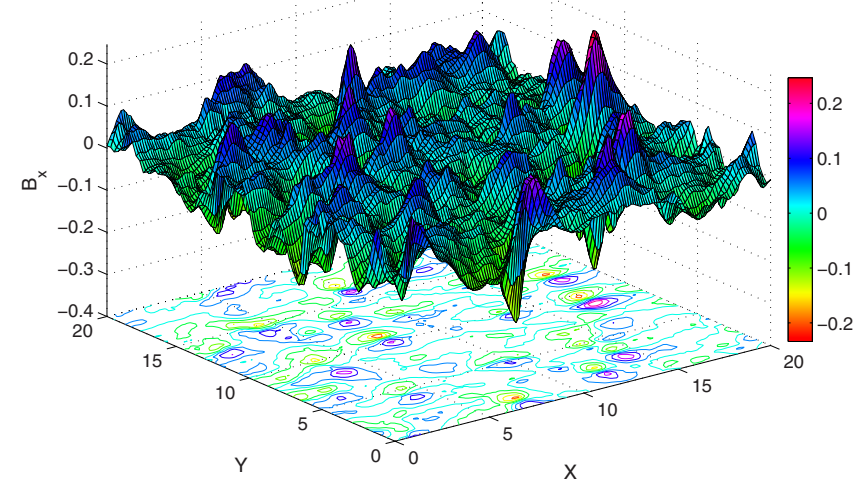

FIG. 9. (Color online) The normalized transverse magnetic field $B_{x}$ at the same time as in Fig. 8. The contour lines on the bottom surface represent planar projection.

$=20 \lambda_{s} \times 20 \lambda_{s} \times 20 \lambda_{s}$. The simulation domain is sampled with a mesh of $160 \times 80 \times 20$ cells. The evolution of the $\mathbf{E}$ and $\mathbf{B}$ fields is done in the same way as in 2D simulations. All other parameters also remain same. Figure 8 shows the structure of the beam filaments during the nonlinear regime at a time $T=16\left(2 \pi / \omega_{p}\right)$ for the simulation case (a). The filaments have high density in the central core surrounded by a low density plasma electron cloud. Figure 9 shows the magnetic fields associated with each filament in the simulation case (a). The filament formation in the simulation case (c) is shown in Fig. 10. In this case, one may recall that in the transverse geometry, the Weibel instability is completely suppressed due to the beam temperature. In longitudinal geometry, some field energy buildup is seen albeit without any significant filamentation. The filamentation of the beam in 3D geometry is clearly seen here now. This filamentation is due to the coupling of the Weibel and two-stream instabilities, which manifests itself into the so-called "oblique mode" [9]. The filamentation of the beam in this case can be attributed to the effective collisionality of the beam-plasma system, arising due to the turbulence caused by the stochastic fields associated with the two-stream instability [19]. Full 3D

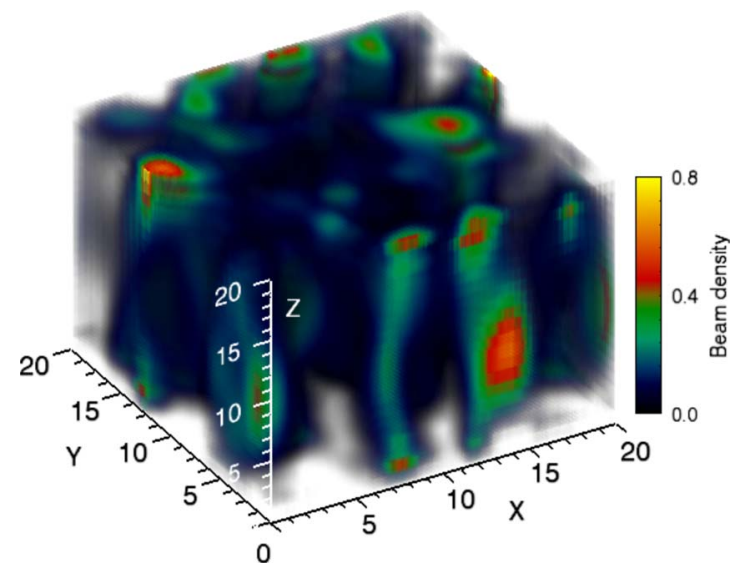

FIG. 10. (Color online) 3D filamentary structures of the electron beam in the simulation case (c), i.e., hot electron beam and collisionless background plasma, at a time $T=10\left(2 \pi / \omega_{p}\right)$. 
simulations for all the other cases have been performed and these are discussed elsewhere [20].

\section{LINEAR ANALYSIS OF THE BEAM-PLASMA SYSTEM}

We have developed a theoretical model based on the Maxwell-fluid system of equations to study the growth rate of the Weibel instability as well as the coupled Weibel-twostream instabilities for the two simulation geometries. The fluid model describes well the linear stage of the instability and has been used to study the Weibel instability in a collisionless as well as collisional plasma recently [10]. In transverse geometry, the Weibel instability is not coupled with the two-stream instability hence can be studied by taking a onedimensional perturbation to linearize the Maxwell-fluid system of equations. However, in longitudinal geometry, the coupling between the Weibel and two-stream instabilities does occur. So we take a 2D perturbation to solve the Maxwell-fluid system of equations. The normalized Maxwell-fluid system of equations is

$$
\begin{gathered}
\boldsymbol{\nabla} \times \mathbf{E}=-\frac{\partial \mathbf{B}}{\partial t}, \\
\boldsymbol{\nabla} \times \mathbf{B}=\frac{\partial \mathbf{E}}{\partial t}+\sum_{a} \mathbf{J}_{a}, \\
\frac{\partial n_{a}}{\partial t}-\boldsymbol{\nabla} \cdot \mathbf{J}_{a}=0, \\
\frac{\partial \boldsymbol{p}_{a}}{\partial t}+\left(\boldsymbol{v}_{a} \cdot \boldsymbol{\nabla} \boldsymbol{p}_{a}\right)=-\left(\boldsymbol{E}+\boldsymbol{v}_{a} \times \boldsymbol{B}\right)-\nu_{e i} \boldsymbol{p}_{a}-\frac{\boldsymbol{\nabla} P_{a}}{n_{a}}, \quad a=p, b,
\end{gathered}
$$

where the subscripts $p$ and $b$ refer to the background plasma and the beam, respectively. All quantities are normalized by the velocity of light, initial plasma density, and plasma frequency.

We begin with a 2D perturbation of the form $\mathcal{F}(x, y, t)$ $=\mathcal{A} \exp \left(i k_{x} x+i k_{y} y-i \omega t\right)$. The electron beam (background plasma) moves in $x(-x)$ direction. For $k_{x}=0$, we get the Weibel instability while for $k_{y}=0$, we get the two-stream instability in the system. For $k_{x} \neq 0$ and $k_{y} \neq 0$, we enter into the regime of so-called oblique mode studied by Bret et al. [9]. As the plasma flow velocity is small, we move to the plasma frame of reference and solve the system of equations in this frame of reference. Since the plasma flow velocity is essentially nonrelativistic, moving to this frame of reference does not affect the magnitude of the growth rate in the laboratory frame of reference. The complete dispersion relation corresponding to the PIC simulations is still lengthy so we write here expressions for the various components of the permittivity of the beam-plasma system,

$$
\begin{gathered}
\epsilon_{x x}=1-\frac{n_{b} k_{x} v_{b}}{\chi \omega \gamma_{b}^{3}}-\frac{n_{b} k_{y}^{2} v_{b}^{2}}{\gamma_{b} \chi \omega^{2}}-\frac{n_{b}}{\gamma_{b}^{3} \omega R_{b}}-\frac{n_{p}}{\omega \beta}, \\
\epsilon_{x y}=-\frac{n_{b} k_{y} v_{b} R_{b}}{\gamma_{b} \omega^{2} \chi},
\end{gathered}
$$

$$
\begin{gathered}
\epsilon_{y x}=-\frac{n_{b} k_{y} v_{b}}{\gamma_{b} \omega^{2} R_{b}}-\frac{3 n_{b} k_{y}^{3} v_{t h}^{2} v_{b}}{\gamma_{b}^{2} \omega^{2} \chi R_{b}}, \\
\epsilon_{y y}=1-\frac{n_{p}}{\omega^{2} \beta}-\frac{n_{b}}{\gamma_{b} \omega^{2}}-\frac{3 n_{b} k_{y}^{2} v_{t h}^{2}}{\gamma_{b}^{2} \omega^{2} \chi},
\end{gathered}
$$

where $n_{b}$ and $n_{p}$ are the density of the electron beam and the background plasma, respectively, $\beta=\left(1+i \widetilde{\nu}_{e i}\right), \quad \widetilde{\nu}_{e i}=\nu_{e i} / \omega$ is the normalized collision frequency, $v_{t h}^{2}=T_{b} / m$ is the square of the electron thermal velocity, $\gamma_{b}=\left(1-v_{b}^{2}\right)^{-1 / 2}, v_{b}$ is the electron beam velocity in the plasma frame of reference, $\chi$ $=\left(\omega-k_{x} v_{b}\right)^{2}-3 v_{t h}^{2} k_{y}^{2} / \gamma_{b}$ and $R_{b}=\left(\omega-k_{x} v_{b}\right)$. In deriving these expressions we have taken $\gamma_{b} \gg 1$, and the background plasma as a cold collisional fluid while the electron beam is a relativistic, hot, and collisionless fluid. The normalized 2D dispersion relation in this case is written as

$$
k_{x}^{2} \epsilon_{x x}+k_{y}^{2} \epsilon_{y y}+k_{x} k_{y}\left(\epsilon_{x y}+\epsilon_{y x}\right)-\omega^{2} \epsilon_{x x} \epsilon_{y y}+\omega^{2} \epsilon_{x y} \epsilon_{y x}=0 .
$$

\section{A. Transverse geometry calculations}

For transverse geometry, we set $k_{x}=0$ in the dispersion relation (6) and solve the dispersion relation numerically in different limits by setting the appropriate values of the collision frequency and the beam temperature. In cases (a) and (b) $\left(\widetilde{\nu}_{e i}=0 \quad v_{t h}=0\right.$ and $\widetilde{\nu}_{e i} \neq 0 \quad v_{t h}=0$, respectively), we recover the same dispersion relations as in Ref. [10]. Similarly we also recover the dispersion relation for the case (c) $\left(\widetilde{\nu}_{e i}\right.$ $\left.=0, v_{t h} \neq 0\right)$. The growth rates of the Weibel instability for these cases are shown in Fig. 11. We see that in the cold collisionless case [subplot (a)], the most unstable mode in the system has characteristic scale length less than the electron skin depth, $\lambda_{s}$. This results in small-scale filament formation. The simulation result [Fig. 1(a)] confirms the smallscale filamentation of the cold beam propagating in a collisionless plasma. The growth rate of the instability varies for small value of $k_{y}$ as $\Gamma \approx k_{y}\left(n_{b} v_{b}^{2} / \gamma_{b}\right)^{1 / 2}$, while it is independent of $k_{y}$ for large value of $k_{y}\left[\Gamma \approx\left(n_{b} v_{b}^{2} / \gamma_{b}\right)^{1 / 2}\right]$, a scaling also observed in Ref. [10].

The influence of collisions in the case of a cold beam is not straightforward. It is seen from Fig. 11(b) where the most unstable mode with wavelength around the plasma skin depth is weakly influenced by small collisions. Its growth rate decreases only slowly with increase in the collision frequency in agreement with the results of Ref. [10]. For large collision frequency, $\widetilde{\nu}_{e i} \gg 1$, it varies as $\widetilde{\nu}_{e i}^{-1 / 2}$. At the same time, the growth rate of the most unstable mode with larger wavelength grows with collisions and is proportional to $\widetilde{\nu}_{e i}^{1 / 2}$. This leads to formation of larger filaments as seen in the simulation [Fig. 1(b)]. The third subplot (c) of Fig. 11 shows the simulation case (c) of the transverse geometry. Evidently, increase in the beam temperature results in the disappearance of the filamentation of the beam. Physically, it can be attributed to the Debye screening. For a beam temperature $T_{b}$ $=70 \mathrm{keV}\left(v_{t h}=0.4\right)$, the Weibel instability is completely suppressed in sync with the simulation results. The case of hot electron beam and cold collisional background plasma is 
(a)

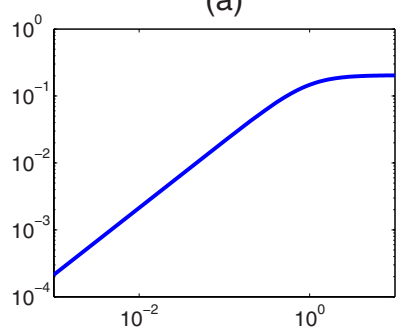

(c)

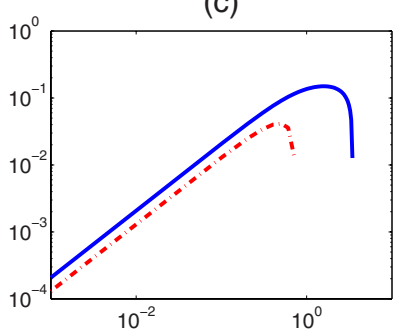

(b)

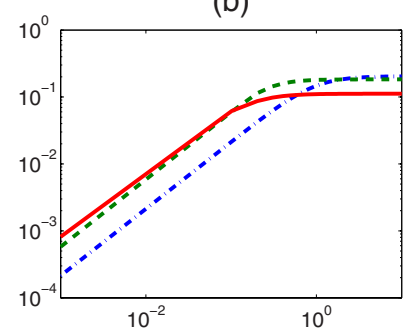

(d)

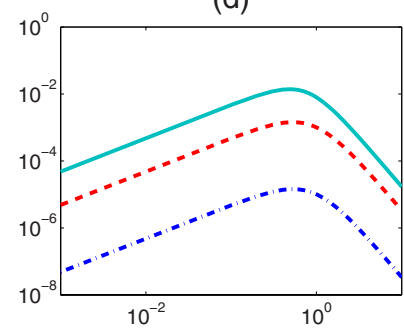

FIG. 11. (Color online) The growth rates calculated from the dispersion relations [Eq. (6)] for the four cases: (a), (b) (dash-dot line, $\widetilde{\nu}_{e i}=0.1$, dash line, $\widetilde{\nu}_{e i}=1$, solid line, $\left.\widetilde{\nu}_{e i}=100\right)$, (c) (solid line, $v_{t h}=0.05$, dash-dot line, $v_{t h}=0.15$ ), and (d) (dash-dot line, $\widetilde{\nu}_{e i}$ $=0.001$, dash line, $\widetilde{\nu}_{e i}=0.1$, solid line, $\widetilde{\nu}_{e i}=1$ ). In all subplots, the vertical axis represents the normalized growth rate, $\Gamma$, and the horizontal axis represents the normalized wave vector, $k_{y}$. The last subplot (d) corresponds to Eq. (7). The other parameters for the beam and plasma are $n_{b}=0.11, v_{b z}=0.92$, and $n_{p}=0.9$, respectively.

fairly complicated for analytical studies due to the higher order (tenth order) of the dispersion relation. So we further simplify the dispersion relation (6) to give a simple dispersion relation in this case. As is the case in our simulations, we assume $n_{p} \gg n_{b}$ and simplify by keeping the lowest order terms in Eq. (6), and write the dispersion relation in a diffusionlike approximation as

$$
\omega^{2} \approx 3 v_{t h}^{2} k_{y}^{2}-\frac{n_{b} k_{y}^{2} v_{b}^{2}}{\gamma_{b}\left(k_{y}^{2}+n_{p} / \beta\right)} .
$$

This dispersion relation is analogous to the one derived by Molvig [21]. The role of the magnetic field which stabilizes the Weibel instability in Molvig's model is replaced here by the beam temperature; which does the same thing as the magnetic field. A plot of the instability growth is shown in Fig. 11(d) for several values of $\widetilde{\nu}_{e i}$ for beam temperature $T_{b}=70 \mathrm{keV}\left(v_{t h}=0.4\right)$. The important result is that even small collisions revive the instability and the growth of the instability initially increases with $k_{y}$ vector and decreases later on after attaining a maximum value. The maximum growth rate increases with collision frequency for small collisions. For higher collision frequency, $\widetilde{\nu}_{e i} \gg \omega_{p}$, the growth will decrease again. Hence, collisions play a different role here. Although the role of collisions in reviving the Weibel instability is mathematically apparent from Eq. (7), it could be understood physically in terms of the unstable negative energy waves. For small collision frequency, the dispersion relation (7) yields three modes. The energy density associated with one of the growing modes can be found to be negative [19]. Thus, we may interpret the collisional Weibel

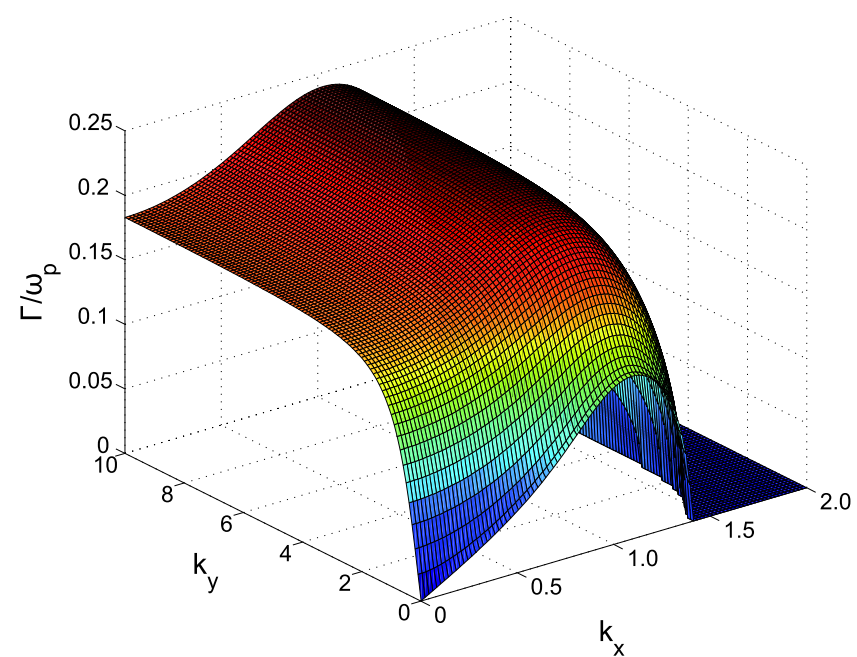

FIG. 12. (Color online) Growth rate variation with normalized $k_{x}$ and $k_{y}$ for the simulation case (a) showing the existence of the oblique mode [9]. The parameters are the same as in other figures.

instability in terms of these unstable negative energy waves. The phenomenon of negative energy wave generation and its consequent effects on the instability have been studied in beam-plasma systems before as well [22,23]. The linear growth rates calculated from theory match well with the simulation results.

\section{B. Longitudinal geometry calculations}

For longitudinal geometry, we retain both $k_{x}$ and $k_{y}$ in Eq. (6) and solve the dispersion relation numerically by setting the appropriate values of parameters as in Sec. II A. Figure 12 shows the growth rate plot with $k_{x}$ and $k_{y}$ in the case (a). One may recover the growth rates of purely Weibel and twostream instabilities if one moves along the $k_{y}$ and $k_{x}$ axes, respectively. The two-stream instability stabilizes for small wave vector $k_{x}$ while the Weibel instability saturates at large value of $k_{y}$. Thus, one may expect to see long scale filaments in $x$ direction while short scale filaments must be formed in $y$ direction. The existence of the oblique mode is evident from the figure, which has been studied in detail by Bret et al. [9]. The oblique mode, which is the most unstable mode, causes beam spraying for finite width beams. Consequently, one may have less energy being deposited in the forward direction. However, for infinite width beam, the oblique mode should not cause much beam spraying. The magnitude of the maximum growth rate is well approximated by the relation [24]

$$
\Gamma=\frac{\sqrt{3}}{2^{4 / 3}}\left(\frac{n_{b}}{n_{p} \gamma_{b}}\right)^{1 / 3} .
$$

For the simulation parameters, the oblique mode makes $\approx 80^{\circ}$ angle with the direction of beam propagation describing the dominance of the Weibel instability over the twostream instability during the linear stage of the filamentation dynamics of the beam. If one looks at the beam density filaments (Fig. 5, top right panel), we see more filaments are being formed in $y$ direction than in $x$ direction, which con- 


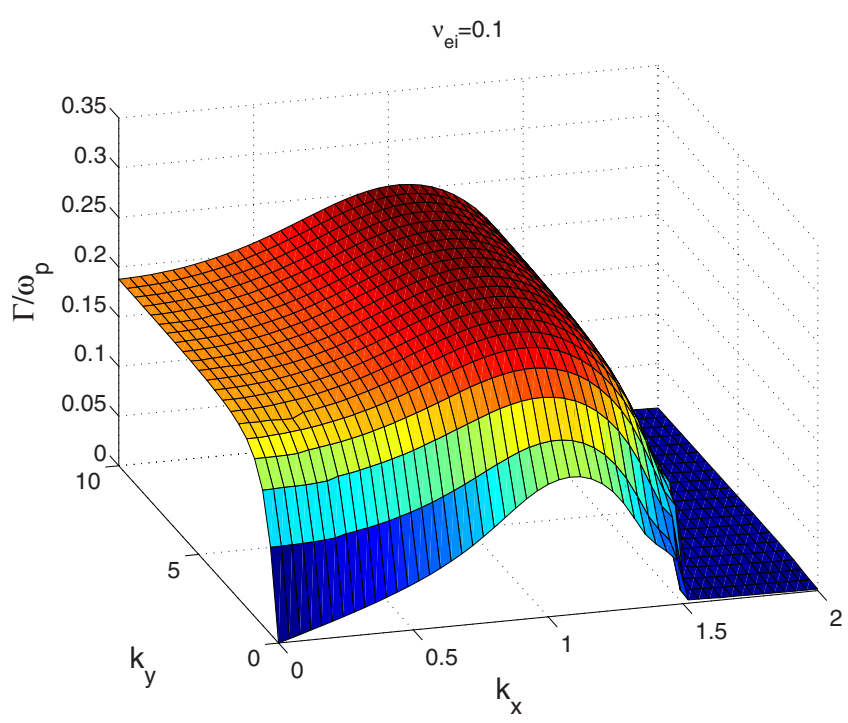

FIG. 13. (Color online) Growth rate map with $k_{x}$ and $k_{y}$ for the simulation case (b). The other parameters are the same as in other figures.

firms the theoretical results. Figure 13 depicts the growth rate in a collisional plasma at normalized collision frequency $\widetilde{v}_{e i}=0.1$. The growth rate map is almost unchanged except the maximum growth rate of the oblique mode is slightly increased. This increase can be attributed to the generation of negative energy waves in the beam-plasma system [22]. Collisions also make the spectrum of the instability slightly broader in $k_{x}$ direction. Hence, stable regions in $k$ space for collisionless two-stream instability become unstable though the growth rate of these regions is significantly small. Due to collisions, the collective effects of plasma come into play and it results in the longer beam filaments formation during the nonlinear stage of the instability. Figure 14 shows the growth rate map in the case (c), where the electron beam has

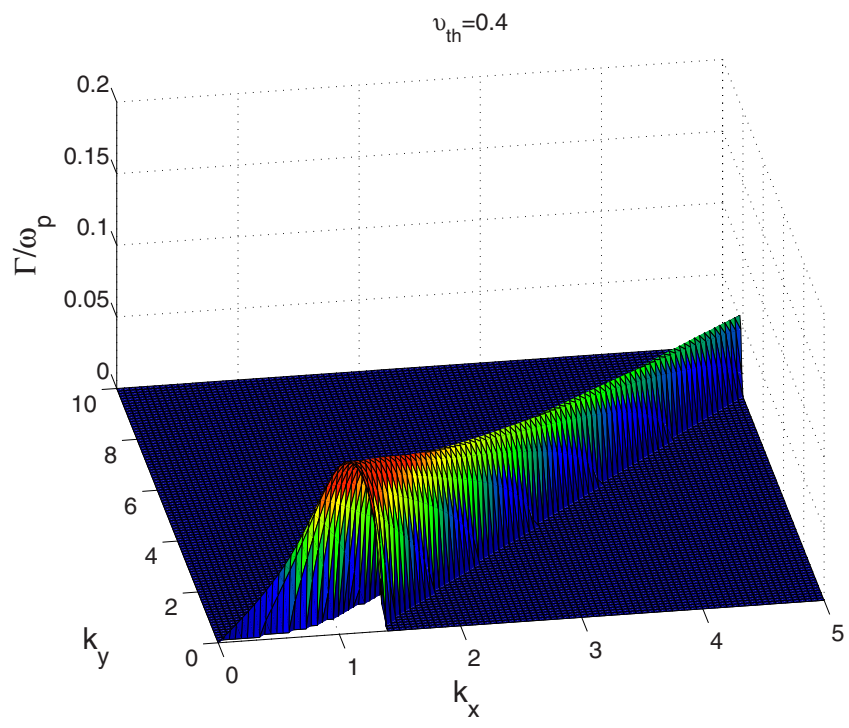

FIG. 14. (Color online) Growth rate map with $k_{x}$ and $k_{y}$ for the simulation case (c). Though the purely Weibel instability is suppressed, the oblique mode is not suppressed.

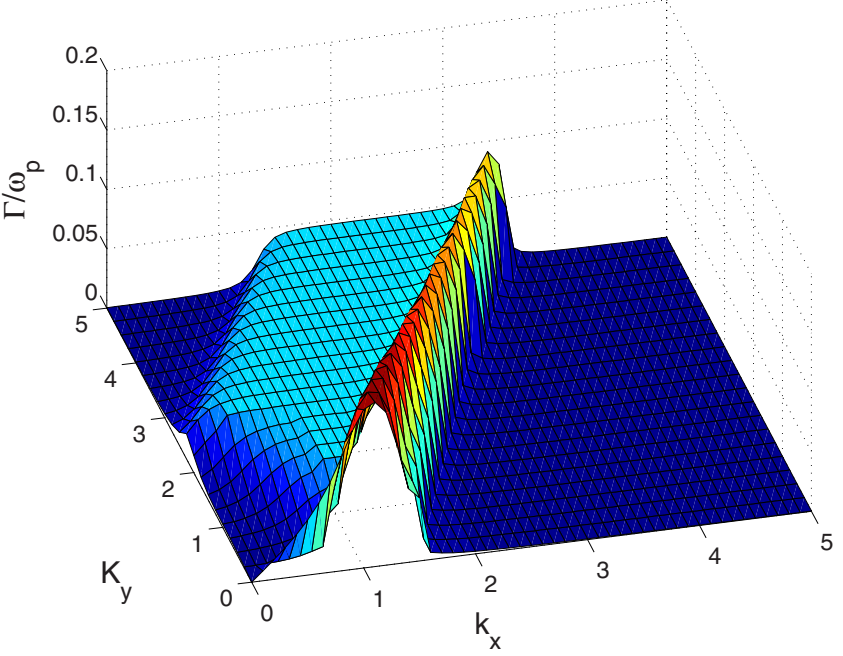

FIG. 15. (Color online) Growth rate map with $k_{x}$ and $k_{y}$ for the simulation case (d). The other parameters are the same as in other figures.

transverse temperature $T_{b}=70 \mathrm{keV}$, which is high enough to suppress the Weibel instability as seen in Fig. 11(c). However, the oblique mode, which is the manifestation of coupling of the Weibel and two-stream modes, is not suppressed; although the growth rate of the instability is significantly reduced. We again notice longer filaments formation in $x$ direction and comparatively small filaments formation in $y$ direction. These results are in agreement with the results obtained by Bret et al. [9]. On inferring from the results of the last section, we may consider that the two-stream instabilityinduced turbulence is providing an effective collisionality to the beam-plasma system, which revives the Weibel instability even though the transverse beam temperature is high enough to suppress the Weibel instability. One may conclude that in 3D geometry, the Weibel instability cannot be suppressed completely due to transverse beam temperature alone. This result is important in the context of FI. Figure 15 depicts the growth rate map for the simulation case (d), i.e., the hot electron beam and collisional background plasma. We observe that collisions make the spectrum of the instability broader. The linear growth rates calculated from theory show good agreement with the simulation growth rates.

\section{CONCLUSIONS}

In summary, we have carried out detailed 2D PIC simulations in two different geometries on the Weibel instability and coupled Weibel-two-stream instabilities of an electron beam, in a parameter regime which is relevant to the FI scheme. Additionally, full 3D PIC simulations have also been performed to understand the filamentation of the beam in a realistic geometry. We have studied the effects of various factors such as electron beam temperature and collisions in the return plasma current separately as well as collectively, thus highlighting the influence of each physical process clearly. The transverse temperature of the electron beam tends to suppress the Weibel instability but collisions in the return plasma current play a paradoxical role and revive back 
the Weibel instability. This paradoxical role of collisions is attributed to the instability of negative energy waves. The coupled Weibel-two-stream instability or the so-called oblique mode remains unsuppressed for significantly high transverse beam temperature. One may treat two-stream instability as a source of an effective collisionality of the beam system, which can drive the filamentation of the hot electron beam in 3D geometry. Thus, the complete suppression of the filamentation instability appears to be difficult in the context of FI. The linear stage of the field energy buildup in simula- tions is found to be in good agreement with the analytical model developed to study the beam-plasma system.

\section{ACKNOWLEDGMENTS}

This work was supported by the DFG through TR-18 project and the U.S. Department of Energy Grants No. DEFG02-04ER41321 and No. DE-FG02-04ER54763. The work of A.K. was partially supported by the Alliance Program of the Helmholtz Association (Grant No. HA216/EMMI).
[1] M. Tabak et al., Phys. Plasmas 1, 1626 (1994).

[2] E. S. Weibel, Phys. Rev. Lett. 2, 83 (1959).

[3] A. Pukhov and J. Meyer-ter-Vehn, Phys. Rev. Lett. 79, 2686 (1997); M. Tatarakis et al., Nature (London) 415, 280 (2002).

[4] M. V. Medvedev and A. Loeb, Astrophys. J. 526, 697 (1999); R. A. Fonseca, L. O. Silva, J. W. Tonge, W. B. Mori, and J. M. Dawson, Phys. Plasmas 10, 1979 (2003); L. O. Silva, R. A. Fonseca, J. W. Tonge, J. M. Dawson, W. B. Mori, and M. V. Medvedev, Astrophys. J. 596, L121 (2003); L. O. Silva, in The Common Physics of AGN, Microquasars, and Gamma-Ray Bursts, AIP Conf. Proc. No. 856 (AIP, New York, 2006), pp. 109-128.

[5] R. Lee and M. Lampe, Phys. Rev. Lett. 31, 1390 (1973).

[6] A. Pukhov and J. Meyer-ter-Vehn, Phys. Rev. Lett. 76, 3975 (1996); Y. Sentoku, K. Mima, S. Kojima, and H. Ruhl, Phys. Plasmas 7, 689 (2000); Y. Sentoku, K. Mima, P. Kaw, and K. Nishikawa, Phys. Rev. Lett. 90, 155001 (2003); O. Polomarov et al., Phys. Plasmas 14, 043103 (2007); L. Gremillet, D. Bénisti, E. Lefebvre, and A. Bret, ibid. 14, 040704 (2007); L. A. Cottrill et al., ibid. 15, 082108 (2008); A. A. Solodov and R. Betti, ibid. 15, 112702 (2008).

[7] L. O. Silva, R. A. Fonseca, J. W. Tonge, W. B. Mori, and J. M. Dawson, Phys. Plasmas 9, 2458 (2002).

[8] P. H. Yoon and R. C. Davidson, Phys. Rev. A 35, 2718 (1987).

[9] A. Bret, M.-C. Firpo, and C. Deutsch, Phys. Rev. E 70, 046401 (2004); Phys. Rev. Lett. 94, 115002 (2005); Phys. Rev. E 72, 016403 (2005); Laser Part. Beams 24, 27 (2006); A. Bret and C. Deutsch, Phys. Plasmas 13, 022110 (2006).

[10] F. Califano, F. Pegoraro, and S. V. Bulanov, Phys. Rev. E 56, 963 (1997); M. Honda, ibid. 69, 016401 (2004).

[11] C. Deutsch, A. Bret, M. C. Firpo, and P. Fromy, Phys. Rev. E 72, 026402 (2005); J. M. Wallace, J. U. Brackbill, C. W. Cran- fill, D. W. Forslund, and R. J. Mason, Phys. Fluids 30, 1085 (1987); T. Okada and K. Niu, J. Plasma Phys. 23, 423 (1980); 24, 483 (1980); W. Kruer, S. Wilks, B. Lasinski, B. Langdon, R. Town, and M. Tabak, Bull. Am. Phys. Soc. 48, 81 (2003).

[12] M. Honda, J. Meyer-ter-Vehn, and A. Pukhov, Phys. Rev. Lett. 85, 2128 (2000).

[13] J. J. Honrubia, M. Kaluza, J. Schreiber, G. D. Tsakiris, and J. Meyer-ter-Vehn, Phys. Plasmas 12, 052708 (2005); J. J. Honrubia, C. Alfonsín, L. Alonso, B. Pérez, and J. A. Cerrada, Laser Part. Beams 24, 217 (2006).

[14] J. J. Honrubia and J. Meyer-ter-Vehn, Nucl. Fusion 46, L25 (2006).

[15] F. Califano, D. Del Sarto, and F. Pegoraro, Phys. Rev. Lett. 96, 105008 (2006).

[16] L. Gremillet, G. Bonnaud, and F. Amiranoff, Phys. Plasmas 9, 941 (2002).

[17] R. Jung et al., Phys. Rev. Lett. 94, 195001 (2005); M. Tatarakis et al., ibid. 90, 175001 (2003); M. Wei et al., Phys. Rev. E 70, 056412 (2004).

[18] A. Pukhov, J. Plasma Phys. 61, 425 (1999).

[19] A. Karmakar, N. Kumar, G. Shvets, O. Polomarov, and A. Pukhov, Phys. Rev. Lett. 101, 255001 (2008).

[20] A. Karmakar, N. Kumar, A. Pukhov, O. Polomarov, and G. Shvets, Phys. Plasmas 15, 120702 (2008).

[21] K. Molvig, Phys. Rev. Lett. 35, 1504 (1975).

[22] R. J. Briggs, Advances in Plasma Physics (Interscience, New York, 1971), Vol. 4, p. 43; H. S. Pécseli, Plasma Phys. 17, 493 (1975).

[23] M. V. Nezlin, Physics of Intense Beams in Plasmas (IOP Publishing, Bristol, 1993), p. 62.

[24] Ya. B. Fainberg, V. D. Shapiro, and V. I. Shevchenko, Sov. Phys. JETP 30, 528 (1970). 